\title{
Pit- and trench-forming osteoclasts: a distinction that matters
}

\author{
Ditte MH Merrild ${ }^{1}$, Dinisha C Pirapaharan ${ }^{1}$, Christina M Andreasen ${ }^{2}$, Per Kjærsgaard-Andersen ${ }^{3}$, Anaïs MJ Møller ${ }^{1}$, \\ Ming Ding ${ }^{2}$, Jean-Marie Delaissé ${ }^{1 *}$ and Kent Søe ${ }^{1 *}$
}

Osteoclasts (OCs) seeded on bone slices either drill round pits or dig long trenches. Whereas pits correspond to intermittent resorption, trenches correspond to continuous and faster resorption and require a distinct assembly of the resorption apparatus. It is unknown whether the distinction between pits and trenches has any biological relevance. Using OCs prepared from different blood donors, we found that female OCs achieved increased resorption mainly through pit formation, whereas male OCs did so through trench formation. Trench formation went along with high collagenolytic activity and high cathepsin $\mathrm{K}$ (CatK) expression, thereby allowing deeper demineralization. A specific CatK inhibitor abrogated the generation of trenches, while still allowing the generation of pits. OCs obtained from bone marrow were more prone to generate trenches than those obtained from blood. Scanning electron microscopy of bone surfaces eroded in vivo showed trenches and pits of similar size as those made by OCs in culture. We conclude that the distinction between trench- and pit-forming OCs is relevant to the differences among OCs from different skeletal sites, different individuals, including gender, and results from differences in collagenolytic power. This indicates a biological relevance and highlights the importance of discriminating between pits and trenches when assessing resorption.

Bone Research (2015) 3, 15032; doi:10.1038/boneres.2015.32; Published online: 1 December 2015

\section{INTRODUCTION}

Bone resorption is performed by osteoclasts (OCs), the only cells known to be capable of resorbing bone.' This property is due to their unique ability to solubilize mineral and collagen, the two main constituents of the bone matrix. Solubilization of mineral is brought about by pumping protons onto the bone surface, thereby generating an acidic environment, whereas collagen degradation is mainly due to activity of cathepsin K (CatK), a cysteine proteinase. ${ }^{2}$ Catk activity and generation of protons have therefore received a lot of attention in the attempt to control the amount of bone degradation and reduce fracture risk.

Importantly, the solubilization of collagen fibers is a more complex event than mineral solubilization, and is by nature a slower process. Furthermore, collagen is not just a substrate to be solubilized by the OC, but also an important antagonist of the polarized resorptive phenotype of the OC. ${ }^{3}$ Therefore, the challenge for the bone resorbing OC is not merely the solubilization of mineral and collagen, but also the synchronization between these two processes.
This need for synchronization was particularly stressed in our recent culture study showing how the resorptive behavior of OCs is affected by inhibitors interfering specifically with the rate of either collagenolysis or demineralization. ${ }^{4}$ It was found that when decreasing the rate of collagenolysis versus demineralization, the OCs make preferentially round excavations, sometimes closely aligned. These excavations were interpreted as short-time resorption events separated by migration periods, i.e., intermittent resorption. ${ }^{5-6}$ They represent the well-investigated "pit" resorption mode, and correspond with OCs showing a circular sealing zone, surrounding the so-called resorption zone, with exocytosis of resorption factors occurring at the periphery and endocytosis of collagen fragments at the center (concentric distribution of endocytosis and exocytosis). ${ }^{7-9}$ In contrast, increasing the rate of collagenolysis versus demineralization leads to a higher proportion of long and deep resorption trenches. ${ }^{4}$ They were interpreted as long periods of continuous resorption where the OC moves while resorbing. ${ }^{10}$ They represent the less

\footnotetext{
'Department of Clinical Cell Biology, Vejle Hospital/Lillebaelt Hospital, Institute of Regional Health Research, University of Southern Denmark, Vejle, Denmark; ${ }^{2}$ Orthopaedic Research Laboratory, Department of Orthopedic Surgery and Traumatology, Odense University Hospital, University of Southern Denmark, Odense C, Denmark and ${ }^{3}$ Department of Orthopedic Surgery, Vejle Hospital/Lillebaelt Hospital, Vejle, Denmark Correspondence: Kent Søe (kent.soee@rsyd.dk)

*These authors contributed equally to this work

Received: 16 September 2015; Revised: 6 October 2015; Accepted: 13 October 2015
} 
well-investigated "trench" resorption mode, and correspond with OCs showing a broad crescent of actin at the leading edge of the moving OC, with exocytosis occurring at the inner rim of the actin crescent, followed by endocytosis further to the rear (aligned exocytosis and endocytosis). ${ }^{8,11}$ The trench-resorption mode is expected to lead to faster resorption, since it is not interrupted by migration episodes as it is the case in the pit-resorption mode. Furthermore, trenches represent a shape which favors bone fragility. ${ }^{12}$

From our inhibitor studies, ${ }^{4}$ it may be anticipated that the levels of Catk activity are an important determinant of the resorption mode and of the aggressiveness of the OCs. Because the levels of CatK activity were reported to vary, depending on where the OC originates from, 2,4, 13-15 we hypothesized that OCs from different origins would have a distinct propensity for generating trenches or pits in accordance with their respective collagenolytic levels. Here we analyze this hypothesis and therefore compare the resorption patterns obtained by culturing human OCs of different origins on bone slices. These comparisons include (i) different tissue origins, i.e., OCs generated from $\mathrm{CD} 14^{+}$human peripheral blood monocytes versus OCs generated from $\mathrm{CD} 14^{+}$human bone marrow (BM) cells; and (ii) interindividual comparisons performed with OCs generated from $\mathrm{CD} 14^{+}$human peripheral blood monocytes of different donors and discriminating for gender. The collagenolytic potential of these respective OC populations were evaluated by using one or several of the following approaches: the levels of the collagen fragment, C-terminal cross-linked telopeptide of type 1 collagen (CTX), in the conditioned medium; ${ }^{16}$ the amount of collagen remaining at the bottom of the excavations; ${ }^{4,17-18}$ the response to a specific CatK inhibitor, ${ }^{4,17-18}$ and the expression levels of CatK. ${ }^{13}$ We also included scanning electron microscopy observations of bone surfaces eroded in vivo, further verifying the in vivo relevance of pits and trenches. Overall, this study aims at evaluating whether the distinction between pit- and trench-making OCs is merely a laboratory peculiarity, or whether it may matter when considering pathophysiological situations.

\section{MATERIALS AND METHODS}

In vitro generation of OCs from human buffy coats Human $\mathrm{CDI}^{+}$monocytes were isolated from buffy coats (BC) donated by 42 healthy female and 30 male volunteers (approved by the local ethics committee, 2007-0019), and differentiated into mature OCs with macrophage colony-stimulating factor (M-CSF) and receptor activator of nuclear factor kappa-B ligand (RANKL) (R\&D Systems, Minneapolis, MN, USA) as previously described. ${ }^{19-20}$ Blood donors are in general between the ages of 18 and 67 years, but their age was not disclosed due to ethical regulation. Informed consent was obtained from all participants. These OCs will be referred to as BC-OCs throughout. For details on the number and gender of the donors used please refer to the figure legends and Supplementary Table S1.

In vitro generation of OCs from human $B M$

Human BM was obtained from four female and seven male osteoarthritis patients with a mean age of 66 years, undergoing hip replacement (approved by the local ethics committee, 2011-0114). Informed consent was obtained from all participants. For details on the number and gender of the donors used please refer to figure legends and Supplementary Table S1. An X-ray image of the femur was used to eliminate those patients with clear signs of osteoporosis at the site of surgery. The small piece of trabecular bone from the femur removed during surgery was handled as previously described. ${ }^{21}$ The washed out $B M$ was resuspended in minimal essential medium $\alpha$ containing 10\% fetal calf serum (FCS; Biological Industries, Kibbutz Beit Haemek, Israel), $25 \mathrm{ng} \cdot \mathrm{mL}^{-1} \mathrm{M}-\mathrm{CSF}$ and RANKL, $2 \mathrm{mmol} \cdot \mathrm{L}^{-1}$ L-glutamine (Sigma-Aldrich, Seelze, Germany), $5 \mathrm{ng} \cdot \mathrm{mL}^{-1}$ transforming growth factor beta 1 (PeproTech, Rocky Hill, CT, USA), and $10 \mathrm{nmol} \cdot \mathrm{L}^{-1}$ dexamethasone (Sigma-Aldrich). Cells were seeded in culture flasks and incubated for $48 \mathrm{~h}$ at $37{ }^{\circ} \mathrm{C}$ and $5 \% \mathrm{CO}_{2}$. Those cells that had not attached to the surface were harvested, resuspended in medium, mixed with warm phosphatebuffered saline (PBS) (1:1), and separated on a FicollPaque PLUS gradient. The interphase was collected, washed in PBS, resuspended in medium, seeded in well plates and incubated for 24-72 h. Medium was very gently removed, in order to not remove the semi-attached cells. The cells were then detached with accutase (PAA, Pasching, Austria), washed in medium, and allowed to recover for $30-60$ min at $4{ }^{\circ} \mathrm{C}$. The $\mathrm{CD} 14^{+}$cells were subsequently isolated as previously described. ${ }^{19-20}$

\section{Bone resorption assays and analyses}

The mature BC-OCs, detached by accutase, and the CDI $4^{+}$BM cells were both seeded on bovine bone slices (IDS Nordic, Herlev, Denmark and Boneslices.com, Jelling, Denmark) at a density of 120000 cells per bone slice, and cultured in the presence of $10 \%$ FCS (Biological Industries), $25 \mathrm{ng} \cdot \mathrm{mL}^{-1} \mathrm{M}$-CSF and RANKL, and supplemented with $2 \mathrm{mmol} \cdot \mathrm{L}^{-1} \mathrm{~L}$-glutamine for the BM cell cultures. When indicated, the specific CatK inhibitor L873724 (a generous gift from Merck, Rahway, NJ, USA) was added to both types of cultures at a concentration of $100 \mathrm{nmol} \cdot \mathrm{L}^{-1}$. L873724 was solubilized in dimethyl sulfoxide (Sigma-Aldrich). This solvent was added at the same amount to the controls, resulting in a final concentration of $0.1 \%(\mathrm{v} / \mathrm{v})$. The number of bone slices used for each condition varied from experi- 
ment to experiment between three and eight bone slices per condition.

In order to enable comparisons of resorption characteristics between BC-OC and BM-OC cultures, the duration of the cultures was calibrated according to the length of the trenches. The rationale for doing so is that the length of a trench reflects active resorption time, so that trenches of a similar length reflect similar durations of resorption. Furthermore, length of trenches in cultures of a given duration was consistent. By performing test cultures of different duration, we found that BM-OCs cultured for 4 days and $\mathrm{BC}-\mathrm{OCs}$ cultured for 3 days generated trenches of similar length.

At culture termination, the cells were removed from the bone slices, resorption events were visualized with toluidine blue (Sigma-Aldrich), and analyzed by light microscopy for the percentage of resorbed surface and resorption depths using a 100-point grid as explained in Boissy et al. ${ }^{19}$ and Soe and Delaisse. ${ }^{20}$ The two types of resorption patterns previously described (pits and trenches) ${ }^{4,20}$ were distinguished in the analysis. A pit was defined as a single excavation, circular in appearance, with well-defined edges, and where the ratio between the length and the width of the excavation did not exceed two. A trench was defined as an elongated and continuous excavation, with welldefined edges and at least two times longer than its width. The total resorbed surface was presented as the percentage of the total bone surface, and the trenches, as percentage of trenches per total resorbed surface. The latter enabled a direct comparison between individual experiments irrespective of variations in the total eroded surface area. The maximum resorption depth was measured before and after removal of the organic material, collagen, from the bone surface, and the thickness of the collagen fringe at the bottom of the excavations was calculated as the difference between these two measurements as previously described. ${ }^{20}$ The investigator was blinded during all analyses.

The levels of CTX, in the conditioned medium was measured as previously described ${ }^{22}$ and in accordance with the supplier's instructions (IDS Nordic, Herlev, Denmark).

\section{Gene expression analyses}

Gene expression analyses were performed on material from experiments with BC-OCs. It was not possible to do this analysis using BM-OCs since it was not possible to obtain enough cells from the same donor to perform both resorption assay and Q-RT-PCR. Mature OCs were generated as described above in the section in vitro generation of OCs from human buffy coats), lyzed, RNA purified (Trizol Plus RNA Purification Kit - Invitrogen, Carlsbad, CA, USA), cDNA produced (iscript kit, Bio-Rad, Hercules, CA, USA), and TaqMan Q-RT-PCR in triplicates were performed as previously described..$^{23}$ The TaqMan primer/probes sets used were; GUS: Hs99999908_ml; Abl: Hs00245443_ml; CatK: Hs00166156_ml; MTl-MMP: Hs01037009_gl; and MMP-9: Hs00234579_ml (Applied Biosystems, Carlsbad, CA, USA). All primer/probe sets were used as instructed by the supplier. Each Q-RT-PCR run was normalized to a CDNA standard curve made each time from the same (randomly chosen) donor. This enabled us to compare between donors and Q-RT-PCR runs. Furthermore, the expression levels of CatK, membrane type 1-matrix metalloproteinase 1 (MT1-MMP), and matrix metalloproteinase 9 (MMP9) were normalized to the average expression level of the house keeping genes, hGUS and hAbl.

\section{Fluorescence microscopy}

Matured BC-OCs were seeded on cortical bone slices $(0.2$ $\mathrm{mm}$ thick) and allowed to resorb for 3 days as described in the section Bone resorption assays and analyses. Cells were fixed with $3.7 \%$ formaldehyde for $15 \mathrm{~min}$, washed with PBS, and stained with fluorescently labeled phalloidin for 20 min. Bone slices were washed in PBS, mounted in ProLong Gold containing 4',6-diamidino-2-phenylindole (Invitrogen), covered with a coverslip, and sealed with clear nail polish. Confocal images were obtained using an Olympus Fluoview FV10i (Olympus Corporation, Shinjuku, Tokyo, Japan) and images were processed using Imaris version 7.6.5 (Bitplan AG, Zurich, Switzerland).

\section{Scanning electron microscopy}

Biopsies were taken from the iliac crest of adult sheep $(\sim 10$ years old; approved by Danish Animal Experiments and Inspectorates, 2011/561-1959) from the study described in Andreasen et al. ${ }^{24}$ The bone samples were fixed in $4 \%$ buffered formaldehyde for $24 \mathrm{~h}$, before transferring to $0.1 \%$ buffered formaldehyde. The biopsies were processed according to a previously published procedure ${ }^{25}$ using a Jeol fine coat gold ion sputter JFC-1100. Scanning electron images were made using a LEO 435VP (Zeiss) operated in a secondary electron mode with a $300 \mathrm{~V}$ positive bias at the detector. The working distance was $35 \mathrm{~mm}$ to assure a large depth of field in the images.

\section{Statistical analyses}

Statistical analyses were performed in GraphPad Prism 4. The types of analyses used are indicated in the figure legends. Significance level was set at $P<0.05$.

\section{RESULTS}

Matching in vitro and in vivo resorption patterns Figure 1 illustrates our definition of pits and trenches, both in vitro and in vivo. Pits are cut perpendicularly to the bone surface by OCs showing a circular actin ring, which surrounds the round hole they are drilling. Trenches are cut 
a

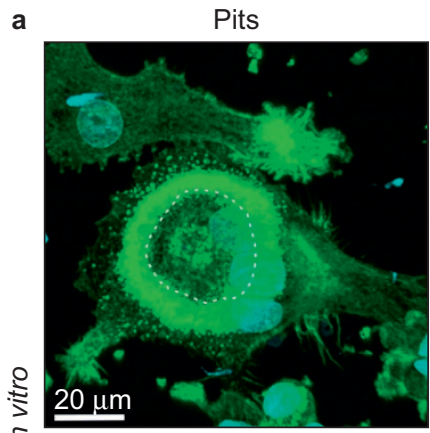

$\leqslant$

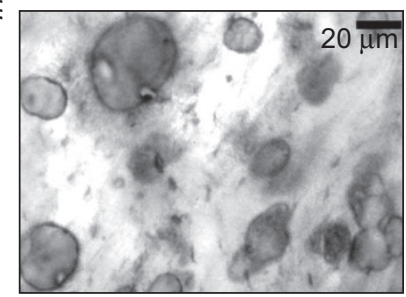

b

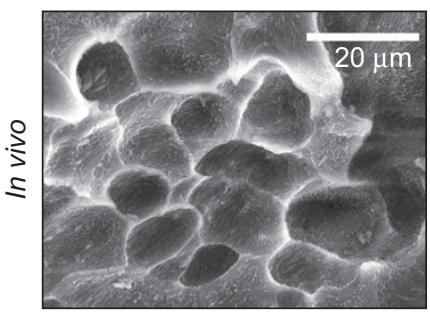

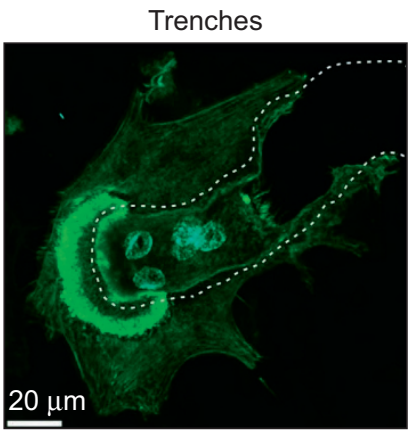

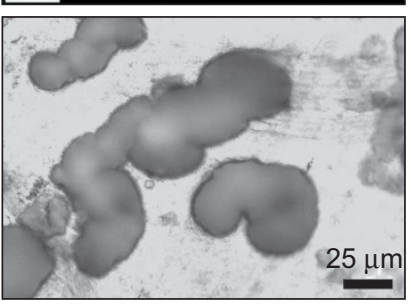

Trenches

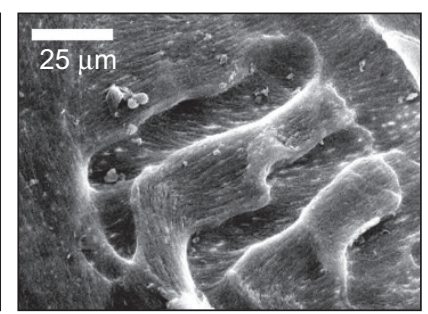

Figure 1. Matching resorption patterns generated by OCs in vitro and in vivo. (a) Pits (left) and trenches (right) generated by OCs in vitro, and corresponding actin configurations of these OCs. Bone slices were exposed to the resorptive activity of BC-OCs. Thereafter, they were either fixed, stained for actin and analyzed through confocal fluorescence microscopy (upper a), or were cleared of OCs, stained with toluidine blue and analyzed through ordinary light microscopy (lower a). Herein, we define a "pit" as a round excavation (lower a) generated by an OC showing a circular actin ring (green) which surrounds the pit (dashed line in upper a), as commonly presented. We define a "trench" as a long excavation reflecting a continuous resorption period progressing along the bone surface (lower a). Trenches are generated by OCs showing a broad crescent of actin (green) polarized at the front edge of the elongating trench (dashed line in upper a). (b) Pits (left) and trenches (right) generated by OCs in vivo. Trabecular bone surfaces from the iliac crest of adult sheep were observed through SEM. The left image shows an area with a resorptive pattern, which closely resembles "pits" observed in vitro, while the right image shows an area with clear signs of continuous resorptive activity closely resembling "trenches" observed in vitro. Please note that the dimensions of pits and trenches observed in vitro and in vivo are comparable (scale bars).

parallel to the bone surface, by OCs showing a crescent of actin polarized at the front of the elongating trench they are digging. ${ }^{11,26}$ As investigated by Mulari et al., ${ }^{26}$ this actin distribution is associated with a distinct organization of the resorption machinery in trench-forming OCs, compared to the commonly investigated pit-forming $O C^{26}$ Trenches are also in line with the mechanism proposed by Stenbeck and Horton ${ }^{10}$ by which OCs simultaneously resorb and progress along the bone surface. Importantly,

pits and trenches also occur in vivo and are of similar size compared to those observed in vitro (Figure 1b), in line with earlier SEM observations. ${ }^{25,27}$

Bone resorption pattern and collagen degradation characteristics of $\mathrm{BM}-\mathrm{OCs}$ and $\mathrm{BC}-\mathrm{OCS}$

Whether derived from BM or BC, OCs seeded on bone slices generated both resorption pits and resorption trenches as typical in other reports. ${ }^{411-12,20,28}$ The proportion of resorbed surface appearing as trenches (or pits) varied considerably depending on the donor (Figure 2a). However, despite this variation, it appeared that BM-OCs were significantly more likely to generate a high proportion of trenches compared with BC-OCs: the extent of trenches reached more than $40 \%$ of the resorbed surface for 10 out of the 11 BM donors, but only for 6 out of the $28 \mathrm{BC}$ donors (Figure 2a). Notably, the difference between the two groups remains highly significant when subdividing them according to the gender of the donors (Supplementary Figure S1).

Of note, trenches were deeper compared with pits (Figure 2b), and in contrast to pits, they showed almost no demineralized collagen at their bottom as reported earlier. ${ }^{4,20}$ This thereby suggests a higher level of collagenolysis in OCs generating trenches than in those generating pits. This held true whether OCs were derived from BC or $\mathrm{BM}$, indicating that greater depths and higher collagenolysis associated with the generation of trenches are independent of the source of OCs. Furthermore, the resorption depth of excavations generated by $B C$ - and $\mathrm{BM}-\mathrm{OCs}$ were in the same range (Figure $2 \mathrm{~b}$ ). This observation is in line with the general model where OCs resorb perpendicularly to the surface until a certain depth (making pits), and continue resorbing laterally (making trenches). ${ }^{4,20}$ Because trenches are not observed below an average depth of $8 \mu \mathrm{m}$, the present data are compatible with the existence of a threshold depth for initiation of trench formation at about a depth of $8 \mu \mathrm{m}$.

Since BM-OCs generated a higher proportion of trenches, and since the generation of trenches reflects a high collagenolytic activity, we searched for quantitative indications that BM-OCs might show a higher collagenoIytic activity. First, we found that resorption pits generated by the 10 tested preparations of BM-OCs showed less demineralized collagen at their bottom, compared with those generated by most of the preparations of BC-OCs (20 tested in total; Figure 2c). Next, we evaluated the efficacy of collagen degradation by measuring the collagen degradation product, CTX, in the conditioned medium and relating it to the extent of resorbed surface in order to determine the efficiency of collagen degradation during erosion. As shown in Figure 2d, this evaluation showed a higher level of collagen degradation products in the 
a

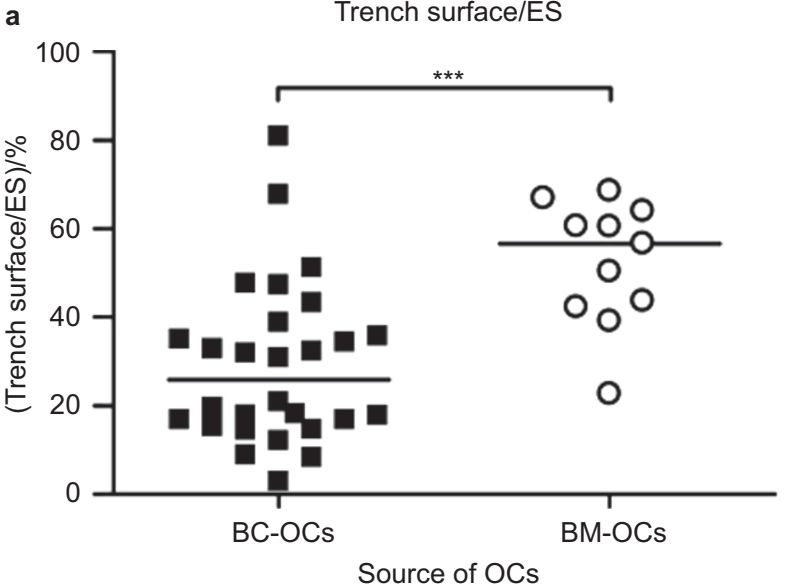

c

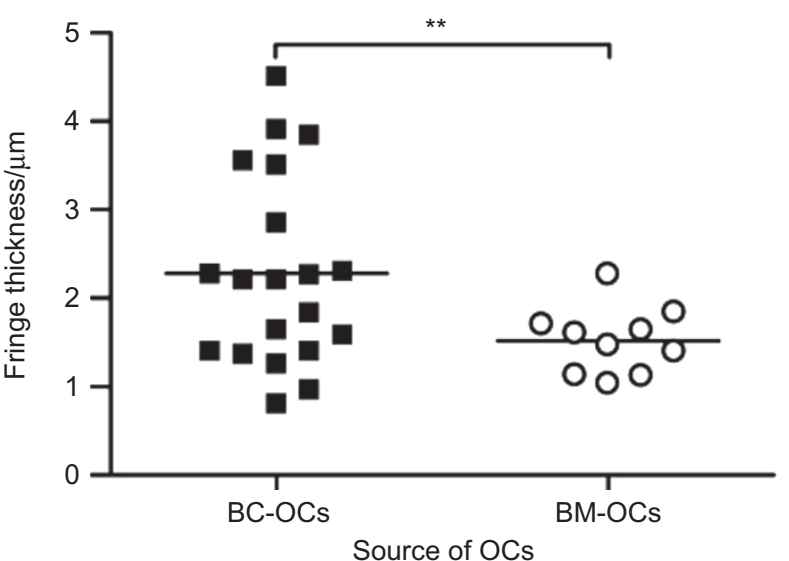

b

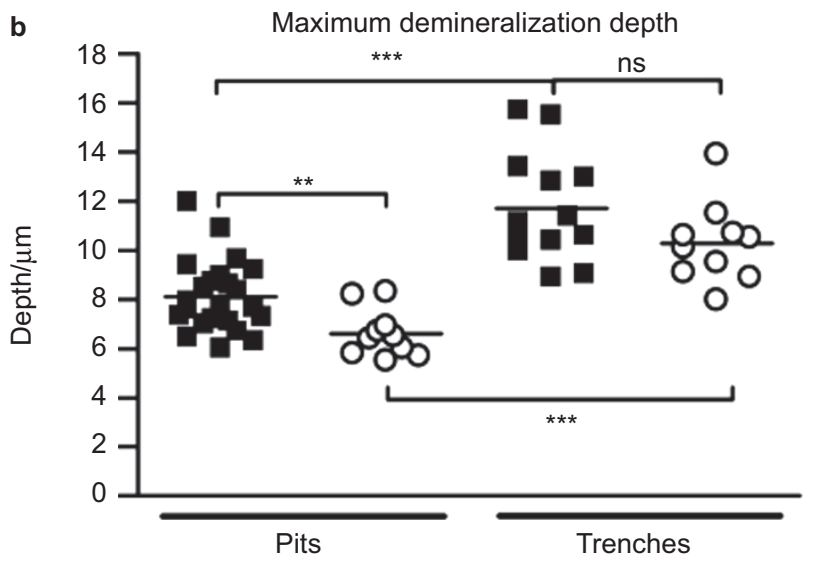

d

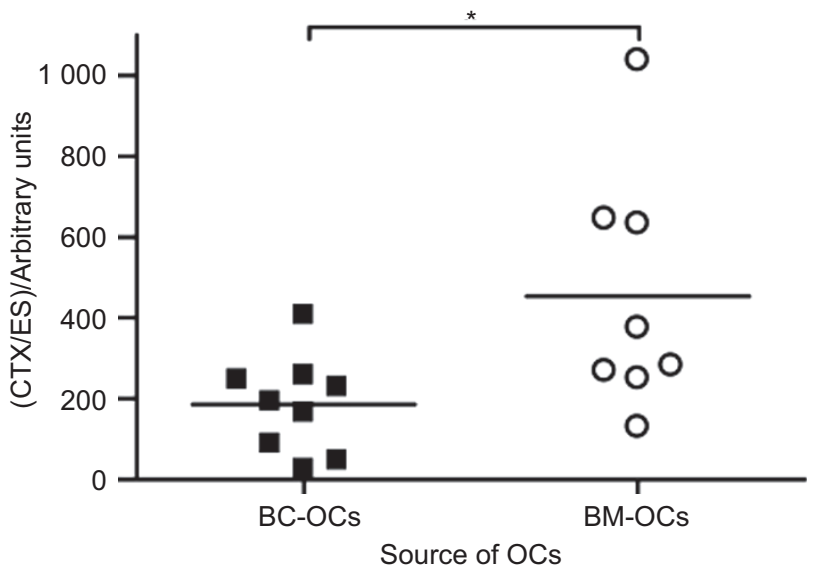

Figure 2. Bone resorption pattern and collagen degradation characteristics of BM-OCs and BC-OCs. BC-OCs and BM-OCs were generated from $n$ different donors and cultured on bone slices (3-8 bone slices per condition for each preparation). These slices were then analyzed for: (a) percentage of trenches per total eroded surface (BC: $n=28, \mathrm{BM}: n=11),(\mathbf{b})$ maximum demineralization depth of pits and trenches $\left(\mathrm{BC}_{\text {pits }}: n=23, \mathrm{BC}_{\text {trenches }}: n=13\right.$, $\left.\mathrm{BM}_{\text {pits: }}: n=10, \mathrm{BM}_{\text {trenches: }} n=10\right)$, (c) thickness of collagen fringe in pits only, as defined in the section Materials and Methods (BC: $\left.n=20, \mathrm{BM}: n=10\right)$, (d) CTX release per eroded surface (BC: $n=9, \mathrm{BM}: n=8)$. The horizontal line in each column indicates the mean $(\mathbf{b}-\mathbf{d})$ or median (a). Statistical analyses: Mann-Whitney test (a) unpaired $t$-test $(\mathbf{b})$ and unpaired $t$-test with Welch correction (c and $\mathbf{d}) . * P<0.05$; ${ }^{* *} P<0.01$; $* * * P<0.001$; ES: eroded surface; ns: not significant.

conditioned medium of BM-OCs, compared with those derived from $B C$.

Thus, taken together, these observations obtained from a large number of independent experiments indicate that a higher propensity to generate resorption trenches goes along with a higher collagenolytic activity during resorption.

Inhibition of collagen degradation affects the resorption pattern of BM and BC-derived OCs

Because the data of the section Bone resorption pattern and collagen degradation characteristics of BM-OCs and $B C$-OCs suggested a relation between generation of trenches and levels of collagenolysis, we investigated whether the formation of trenches would be prevented by inhibiting CatK, which is highly expressed in OCs ${ }^{29-30}$ and is the most potent collagenolytic proteinase known amongst all mammalian proteinases. ${ }^{31}$ Earlier observations on BC-OCs have already shown that prevention of collagen degradation almost abolishes the formation of trenches, ${ }^{4}$ but it had still to be investigated whether this holds true for BM-OCs, which have a higher propensity to generate trenches. Figure $3 a$ shows that whatever the level of trenches in control conditions, inhibition of CatK brings the generation of trenches down to between 0 and $10 \%$ of the resorbed surface. This observation shows that Catk-driven collagen degradation is an absolute requirement for the formation of trenches. From a technical point of view, this observation also shows that the inhibitor concentration used in these experiments is sufficient to almost completely prevent this event, whatever its magnitude.

Resorption depth is another excavation-shape parameter commonly believed to be affected by the rate of collagen degradation. Our earlier BC-OC experiments were analyzed without distinguishing between the depths 
of pits and trenches. ${ }^{4}$ They showed that a general cysteine proteinase inhibitor rendered excavations almost half as deep, in accordance with published knowledge, ${ }^{2,32-34}$ However, only pits are present in inhibitor-treated conditions, whereas both pits and trenches are present in control conditions (Figure 3a). When now taking only pits into account, we found that a specific Catk inhibitor decreased the resorption depths by only $14 \%$ in 8 BC-OC preparations, and by $12 \%$ in $8 \mathrm{BM}-\mathrm{OC}$ preparations (Figure 3b). This shows that the main contribution of CatK to overall excavation depth is by inducing trenches, which prove to be deeper than pits (Figure 2b). However, it is interesting to note that this weak effect (Figure 3b) is enough to bring pit depths below the putative threshold (on average) of about $8 \mu \mathrm{m}$ for trench formation (Figure 2b). The prevention of trench formation by Catk inhibition may thus result at least in part from a decreased excavation depth.

We also checked whether an endpoint more directly related to collagen degradation was affected by CatK inhibition in these experiments. As expected, CTX levels per resorbed surface were decreased in conditioned media from both $\mathrm{BC}-\mathrm{OCs}$ and $\mathrm{BM}-\mathrm{OCs}$, indicating that overall resorption proceeded with less efficient collagen degradation (Figure 3c).

Taken together, these observations strengthen our previous conclusion that CatK-driven collagen degradation is mandatory for the generation of resorption trenches. ${ }^{4}$ This

a Trench surface/ES
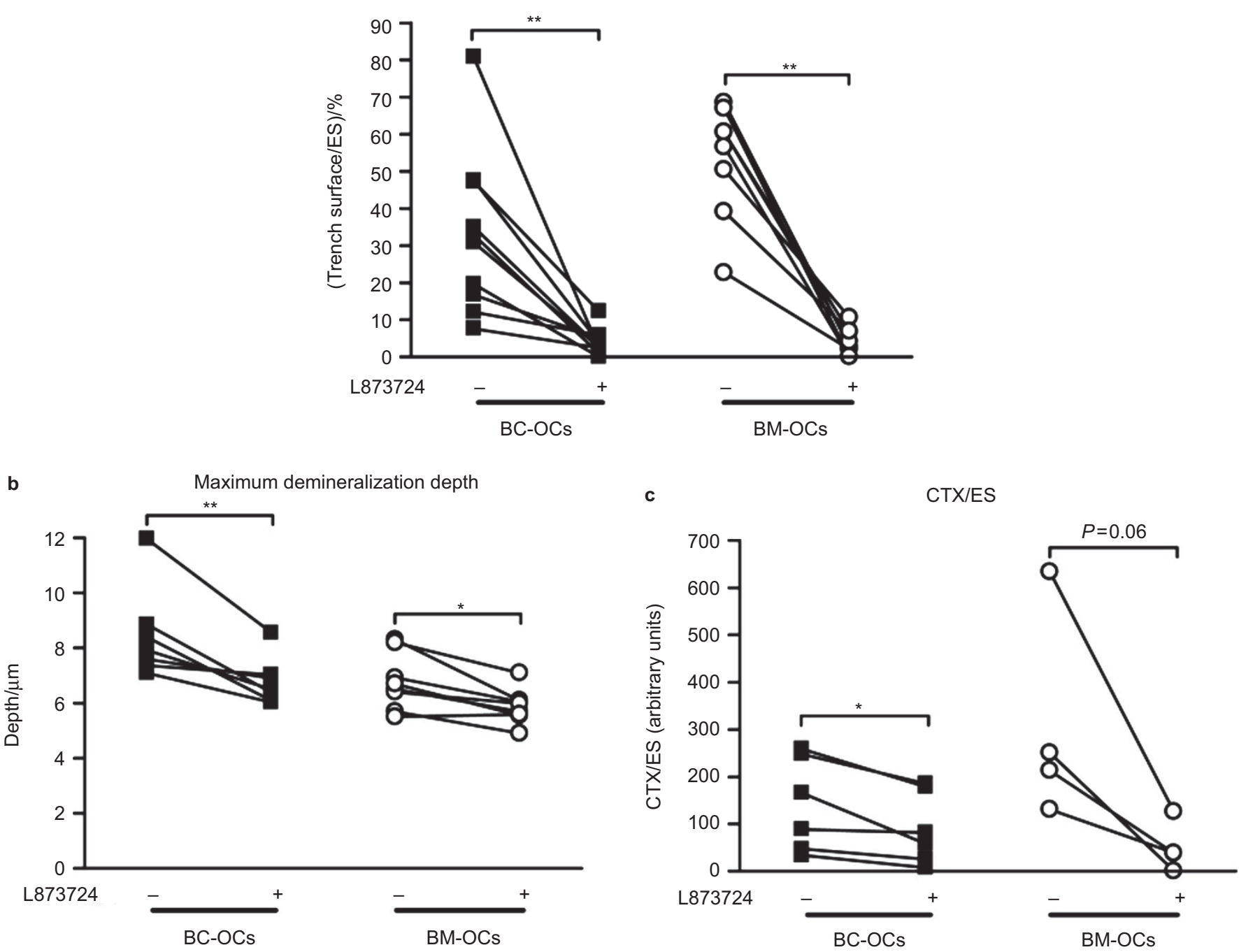

Figure 3. Inhibition of collagen degradation affects the resorption pattern of BM and BC-derived OCs. BC-OCs (black squares) and BM-OCs (open circles) were generated from $n$ different donors and cultured in the absence (-) or presence (+) of the specific CatK inhibitor $\mathrm{L}_{873724}\left(100 \mathrm{nmol} \cdot \mathrm{L}^{-1}\right)$ on bone slices (3-8 slices per condition and preparation). The bone slices were analyzed for: (a) percentage of trenches per total eroded surface (BC: $n=10$, BM: $n=8),(\mathbf{b})$ maximum demineralization depths of pits only (BC: $n=8, \mathrm{BM}: n=8)$, and (c) CTX release adjusted for eroded surface (BC: $n=6$, BM: $n=4)$. Statistical analyses: Wilcoxon matched pairs test. 
a

Trench surface/ES

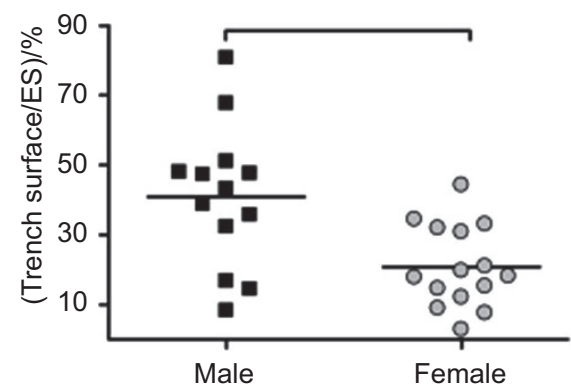

b

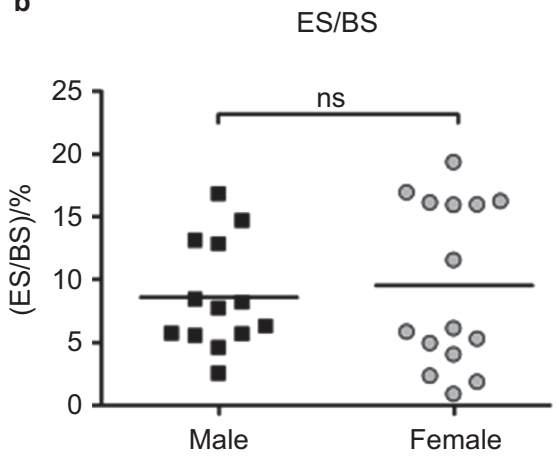

c
Maximum demineralization depth

Gender effect

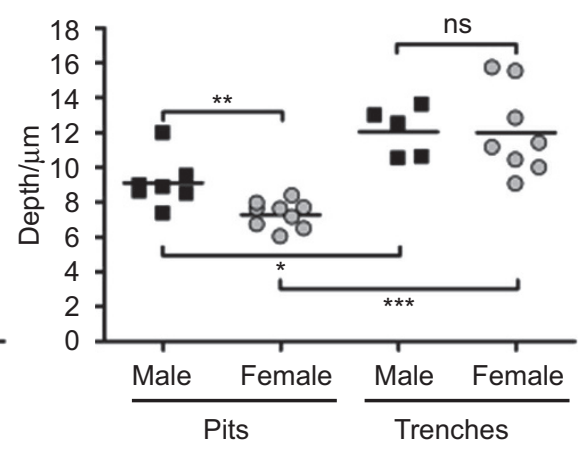

d

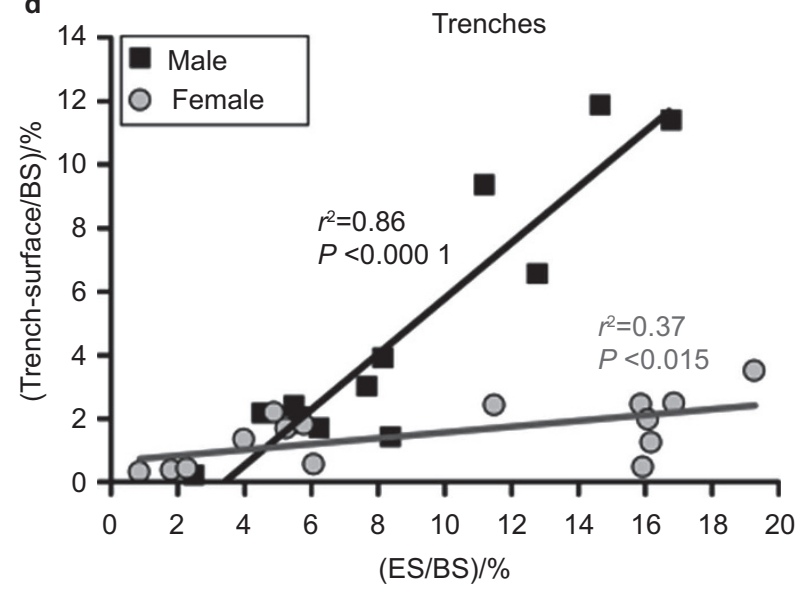

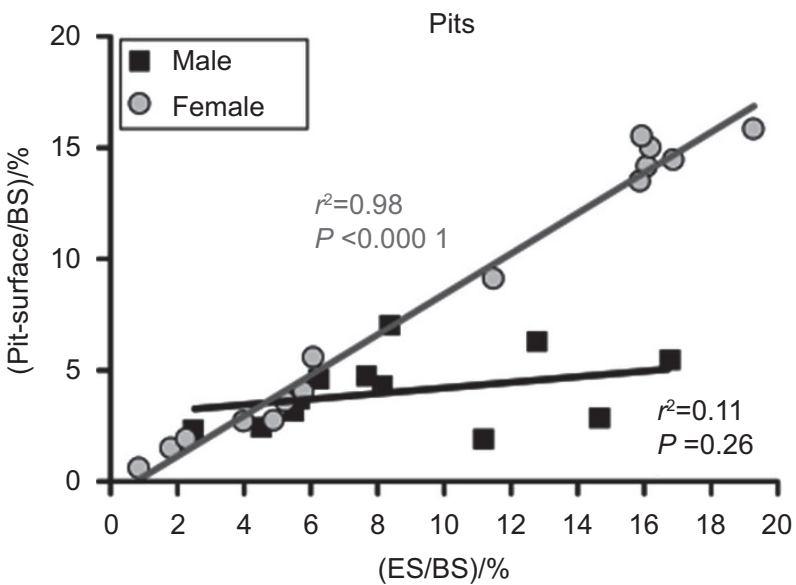
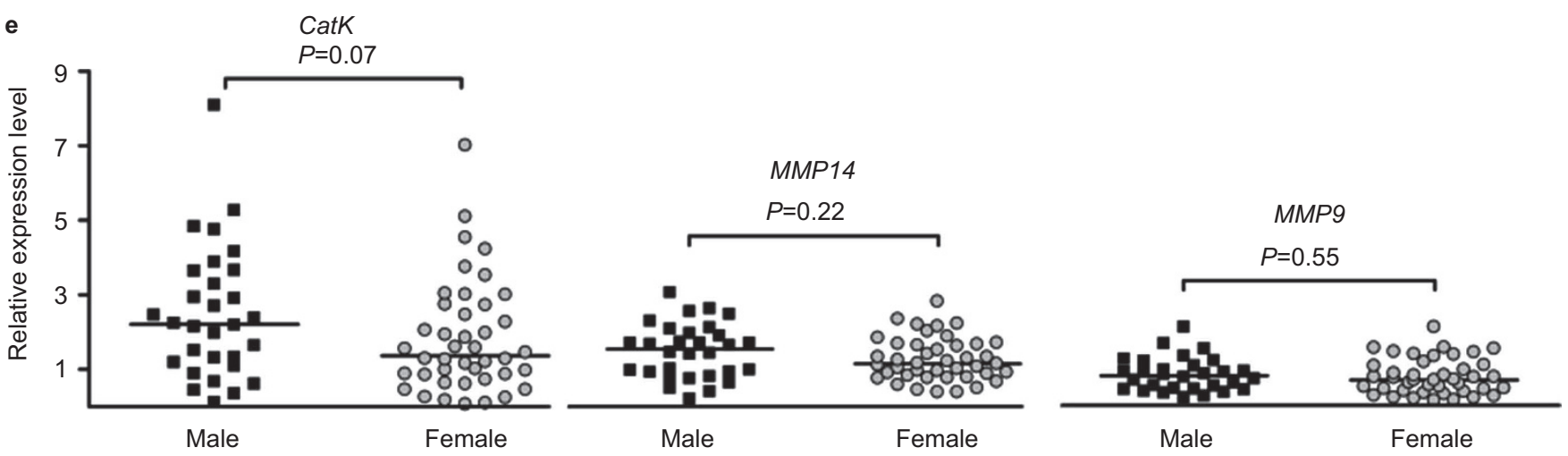

Figure 4. Comparison of male and female BC-OCs with respect to generation of pits and trenches. BC-OCs from male and female donors were generated and cultured on bone slices (3-8 bone slices for each preparation). (a) Impact of the gender on the generation of trenches (BC-male: $n=$ 13, BC-female: $n=15)$. (b) Impact of the gender on the generation of eroded surface (BC-male: $n=13$, BC-female: $n=15)$. (c) Impact of the gender on the maximum resorption depth of pits and trenches (pits $\mathrm{BC}_{\text {male }}: n=7, \mathrm{BC}_{\text {female }}: n=9$ ); trenches $\mathrm{BC}_{\text {male }}: n=5, \mathrm{BC}_{\text {female: }} n=8$ ). (d) Impact of the gender on the relationship between the eroded surface/bone surface and the extent of trench surface/bone surface (left graph) or pit surface/bone surface (right graph) (male: $n=13$, female: $n=15)$. Correlations and $P$ values are indicated in the graphs. (e) Gene expression levels of CatK, MMP14, and MMP9 in BC-OCs subdivided according to the gender of the donor (male: $n=30$, female: $n=42)$. Statistical analyses: Mann-Whitney test (a, $\mathbf{b}$, c, and e), and linear correlation (Pearson) (d). BS: bone surface.

conclusion was recently confirmed by others, ${ }^{28}$ and is in line with other reports. ${ }^{35}$ Furthermore, the present observations highlight that inhibition of collagen degradation has a bigger impact on the OCs that resorb bone according to the trench pattern, compared to those that resorb bone mostly according to the pit pattern. It is therefore expected that the pharmacological administration of Catk inhibitors may affect bone resorption differently depending on the levels of CatK at different bone sites or in different individuals. 
Comparison of male and female BC-OCs with respect to generation of pits and trenches

Figure $2 a$ showed the considerable interindividual variation in the proportion of resorbed surface appearing as trenches, depending on the donors. Figure 4 a shows that this interindividual variation, at least in part, depends on the gender of the donor. Male BC-OCs erode far more surface as trenches compared with female BC-OCs. This gender effect is strictly on the resorption mode, and is not seen on the total eroded surface (Figure $4 \mathrm{~b}$ ). This indicates a different contribution of trenches and pits to the eroded surface, depending on whether erosion is generated by male or female OCs. In order to get insight in these respective contributions, we plotted trench and pit surface against eroded surface (Figure 4d). These plots show that male OCs achieve increased erosion by generating more trench surface - but not more pit surface - and conversely that female OCs achieve increased erosion by generating more pit surface - but not more trench surface. With respect to resorption depth, Figure $4 c$ shows that trenches are on average around $12 \mu \mathrm{m}$ deep for both genders. However, the depth of pits generated by male BC-OC resorb is on average $9 \mu \mathrm{m}$, which is significantly deeper compared with the average of $7 \mu \mathrm{m}$ reached by female BC-OCs. These respective depth values further support the hypothesis that a threshold depth of about $8 \mu \mathrm{m}$ is a prerequisite for trench formation, as mentioned above.

Our results thus clearly show the interest of separately quantifying pits and trenches, and not limiting the measurements to eroded surface. Furthermore they show that one should pay attention to gender when performing osteoclastic resorption experiments.

The reason for the difference between male and female OCs does not relate to differences in fusion potential of male and female OC precursors because OCs from both genders contain the same number of nuclei per $\mathrm{OC}$ at the end of the culture (Supplementary Figure S2). However, one may speculate that the reason relates to CatK levels, since a high propensity to generate trenches goes along with high collagenolysis (Figures 2 and 3). ${ }^{4,20}$ Therefore, we compared male and female OCs from a large cohort for their level of Catk expression, as well as for two other proteinases highly expressed in OCs, MMP14 (also called
MT1-MMP), and MMP9. The expression level of CatK proved to be highly variable $(\mathrm{max} / \mathrm{min}=146: 1)$ for both genders, but a clear trend $(P=0.07)$ also suggests that there on average is a higher expression of CatK in OCs generated from male donors than in those generated from female donors (Figure 4e). In contrast, there are no differences between genders in the expression levels of other prominent collagenolytic proteases such as MMP 14 $(P=0.22)$ and MMP9 $(P=0.55)$ and also their levels of variation are far less compared to CatK (MMP14: $\mathrm{max} / \mathrm{min}=$ 16:1; MMP9: 17:1).

\section{DISCUSSION}

The resorption behavior of OCs is routinely investigated on OCs cultured on bone slices, and most effort was devoted to explain the formation of "pits", i.e., round holes in the bone surface. The commonly accepted model describes alternating periods where the $\mathrm{OC}$ attaches to the bone to resorb perpendicularly to the bone surface, and periods where the OC detaches to migrate over the bone surface without resorbing. However, a few reports indicate that cultured OCs are also able to resorb while migrating, $, 8,10$ thereby generating trenches which reflect long periods of resorption parallel to the bone surface. This "trench" resorption mode did not receive much attention, despite its very contrasting characteristics compared with the "pit" resorption mode (Table 1). The trench mode was recently proposed to involve high rates of collagenolysis versus demineralization, as investigated through pharmacological manipulation. ${ }^{4}$ The present study shows that the distinction between the trench- and pit-resorption mode is relevant to the natural variations found in vivo and in human OCs of different origin cultured on bone slices. Furthermore, it shows that this distinction is systematically associated with differences in the levels of collagenolysis or of Catk expression, thus supporting the critical role of Catk in trench formation.

First, we draw the attention on the resemblance between in vivo resorption patterns and the pits and trenches generated in culture. Variations in the shape of the excavations made by OCs in vivo have been known for a long time, ${ }^{36}$ but they had never been ascribed to possible distinct resorption modes of the OCs, as the

Table 1. Hallmarks of pit- and trench-forming OCs

\begin{tabular}{llll}
\hline Osteoclastic hallmark & Pits & Trenches & References \\
\hline $\begin{array}{l}\text { Movement } \\
\text { Symmetry of resorption apparatus }\end{array}$ & NO & YES & \\
- Actin & Ring & Crescent-like & {$[4,8,10,20]$} \\
$\begin{array}{l}\text { - Exocytosis } \\
\text { - Endocytosis }\end{array}$ & Peripheral & Front side & {$[8,11]$} \\
Balanced collagen \& mineral clearance & Central & Back side & {$[8]$} \\
Cavitation depth & NO (most often) & YES & {$[8]$} \\
\hline
\end{tabular}


existence of distinct resorption modes had not yet been recognized. Of note, Parfitt ${ }^{37}$ inferred from observations on bone sections, that OCs in vivo are often likely to resorb bone parallel to the bone surface. Full understanding of in vivo bone remodeling may thus require more attention for the poorly investigated trench mode, rather than for the pit mode.

Natural variations between the OC populations generated from different blood donors are well known by bone scientists because they appear as a technical problem rendering experimental reproducibility difficult to achieve when working on human material. However, this variation is usually not addressed, even if it is of potential interest in relation with differences in fracture risk, outcome of bone disease, and response to anti-resorptive treatment among different patients. Herein we extend this natural variation between donors to the prevalence of trenches, indicating that some patients may be subjected to a more aggressive type of resorption than others. A remarkable finding is that male OCs generate more trench surface, not pit surface, when eroding more bone, whereas female OCs generate more pit surface rather than trench surface when eroding more bone. This finding is in line with earlier observations showing a higher resorptive activity in male OCs, as assessed in vitro. ${ }^{38}$ Furthermore, serum CTX levels are significantly higher in young healthy adult males compared to young healthy adult females, ${ }^{39-41}$ while serum TRACP5b levels, a marker of OC number, do not appear to be significantly different. ${ }^{41-42}$ This observation may reflect higher CatK activity in OCs of males compared to females also in vivo. It is presently not clear how this gender difference may relate to previously reported gender differences in osteoclastic gene expression, but TNF-related apoptosis-inducing ligand may be a factor that deserves further investigation in this aspect. ${ }^{43}$

Natural variations among OCs from different skeletal sites have also been repeatedly reported. ${ }^{44-45}$ Differences between flat and long bone OCs have been particularly highlighted with respect to response of resorption to pharmacological agents and to gene defects. ${ }^{2,14-15,46}$ Of interest in respect with the present study is that long bone OCs are richer in CatK than flat bone OCs. Accordingly, deletion or pharmacological inhibition of CatK affected more drastically resorption of long bones than that of flat bones. In contrast, resorption of flat bones but not that of long bones was slowed down by inhibitors of MMPs, weaker collagenolytic proteinases believed to contribute to collagen degradation in situations where CatK is deficient. However, these earlier studies did not consider whether these differences in collagenolytic pathways affect the resorption mode of the OCs, i.e., whether they affect the resorption pattern. Herein, we show that the stronger collagenolytic power of BM-OCs compared to BC-OCs goes along with a higher likelihood of generating a higher proportion of trenches compared to pits. We show that this great generation of trenches is also completely dependent on CatK activity, since the addition of a specific CatK inhibitor abrogates their generation. In contrast, this CatK inhibitor still allows pit formation. CatK inhibitors thus have a stronger impact on OCs exerting aggressive resorption. The mechanism relating CatK activity to trench formation has been extensively discussed in Soe et al. ${ }^{4}$ and Soe and Delaisse. ${ }^{20}$ In addition, it is of interest to note that the unique sub-osteoclastic topography created by high collagenolysis ${ }^{20}$ may lead to stabilizing the actin ring, ${ }^{47}$ thereby allowing continuous resorption.

An interesting issue is whether the prevalence of a given resorption mode is due to different precursor origins showing distinct genetic characteristics, or to the response of the same OCs to differences in the environment or levels of systemic regulators. As discussed elsewhere, ${ }^{44-45}$ there are indications that both reasons may be involved. The importance of genetic characteristics is clearly supported by the present findings that the gender predisposes to the erosion mode, i.e., trenches or pits. Regarding the heterogeneity resulting from environmental conditions, it is of interest that CatK expression and the rate of collagenolysis can to some extent be modulated independently from the rate of demineralization, for example, through glucocorticoids $^{20}$ (see discussion in Refs. ${ }^{4,20}$ )

We are aware that this study has some drawbacks due to the mostly retrospective nature of the study. We have not been able to age-match the cohorts, since we did not have an ethical approval to obtain the age of the blood donors. However, in the study of Jevon et al., ${ }^{38}$ it was demonstrated that there was no difference in the extent of resorption by $\mathrm{BC}$-OCs obtained from donors that were younger than 50 years and those that were older. We have not been able to gender-match BC-OCs and BMOCs. However, Supplementary Figure S1 shows that the difference between $\mathrm{BC}-\mathrm{OCs}$ and $\mathrm{BM}-\mathrm{OCs}$ with respect to trench surface per eroded surface is also highly significant when including the differences in the proportion of males and females in the two cohorts into the analysis.

In conclusion, the present study indicates that the classification of cavitations into pits and trenches is relevant to the in vivo resorption patterns, to the natural differences between different $O C$ populations, including gender, and to the levels of their collagenolytic power. Catk is mandatory for continued resorption leading to trenches - an information which may be critical when considering Catk inhibitors such as odanacatib as anti-osteoporotic drugs. ${ }^{48}$ The mechanism driving trench-forming OCs deserves attention in future research. However, whatever the mechanism is, these findings already clearly demonstrate the great importance of discriminating between pits and trenches as well as the gender of donors when 
quantifying erosion in the classical $\mathrm{OC}$ resorption assays performed on bone or dentine slices.

\section{Acknowledgements}

We would like to thank Jacob Bastholm Olesen and Vibeke Nielsen for excellent technical assistance and Merck\&Sharp\&Dohme for providing and granting us the use of the specific CatK inhibitor L873724. Moreover, we would also like to thank the project nurse, Jane Schwartz Leonhardt, at the Department of Orthopedic Surgery at Vejle Hospital for her great collaboration. We also wish to thank associate professor Per Morgen (Department of Physics, Chemistry and Pharmacy, University of Southern Denmark) for friendly assistance and granting us access to the scanning electron microscope. This study was financed by Vejle Hospital/Lillebaelt Hospital.

\section{Competing interests}

The authors declare no conflict of interest.

\section{References}

1 Delaissé JM, Andersen TL, Engsig MT, Henriksen K, Troen T, Blavier L. Matrix metalloproteinases (MMP) and cathepsin K contribute differently to osteoclastic activities. Microsc Res Tech 2003; 61: 504-513.

2 Saftig P, Hunziker E, Wehmeyer O et al. Impaired osteoclastic bone resorption leads to osteopetrosis in cathepsin-K-deficient mice. Proc Natl Acad Sci U S A 1998; 95: 13453-13458.

3 Takahashi N, Ejiri S, Yanagisawa S, Ozawa H. Regulation of osteoclast polarization. Odontology 2007; 95: 1-9.

4 Søe K, Merrild DM, Delaissé JM. Steering the osteoclast through the demineralization-collagenolysis balance. Bone 2013; 56: 191-198.

5 Lakkakorpi PT, Väänänen HK. Kinetics of the osteoclast cytoskeleton during the resorption cycle in vitro. J Bone Miner Res 1991; 6: 817-826.

6 Saltel F, Destaing O, Bard F, Eichert D, Jurdic P. Apatite-mediated actin dynamics in resorbing osteoclasts. Mol Biol Cell 2004; 15: 5231-5241.

7 Hirvonen MJ, Fagerlund K, Lakkakorpi P, Väänänen HK, Mulari MT. Novel perspectives on the transcytotic route in osteoclasts. Bonekey Rep 2013; 2: 306.

8 Mulari MT, Zhao H, Lakkakorpi PT, Väänänen HK. Osteoclast ruffled border has distinct subdomains for secretion and degraded matrix uptake. Traffic 2003; 4: 113-125.

9 Salo J, Lehenkari P, Mulari M, Metsikkö K, Väänänen HK. Removal of osteoclast bone resorption products by transcytosis. Science 1997; 276: 270-273.

10 Stenbeck G, Horton MA. A new specialized cell-matrix interaction in actively resorbing osteoclasts. J Cell Sci 2000; 113: 1577-1587.

11 Rumpler M, Würger T, Roschger P et al. Osteoclasts on bone and dentin in vitro: mechanism of trail formation and comparison of resorption behavior. Calcif Tissue Int 2013; 93: 526-539.

12 Vanderoost J, Søe K, Merrild DM, Delaissé JM, van Lenthe GH. Glucocorticoid-induced changes in the geometry of osteoclast resorption cavities affect trabecular bone stiffness. Calcif Tissue Int 2013; 92: 240-250.

13 Everts V, Korper W, Jansen DC et al. Functional heterogeneity of osteoclasts: matrix metalloproteinases participate in osteoclastic resorption of calvarial bone but not in resorption of long bone. FASEB J 1999; 13: 1219-1230.

14 Everts V, Korper W, Hoeben KA et al. Osteoclastic bone degradation and the role of different cysteine proteinases and matrix metalloproteinases: differences between calvaria and long bone. J Bone Miner Res 2006; 21: 1399-1408.
15 Gowen M, Lazner F, Dodds R et al. Cathepsin K knockout mice develop osteopetrosis due to a deficit in matrix degradation but not demineralization. J Bone Miner Res 1999; 14: 1654-1663.

16 Garnero P, Ferreras M, Karsdal MA et al. The type I collagen fragments ICTP and CTX reveal distinct enzymatic pathways of bone collagen degradation. J Bone Miner Res 2003; 18: 859-867.

17 Everts V, Delaissé JM, Korper W, Niehof A, Vaes G, Beertsen W. Degradation of collagen in the bone-resorbing compartment underlying the osteoclast involves both cysteine-proteinases and matrix metalloproteinases. J Cell Physiol 1992; 150: 221-231.

18 Everts V, Delaissé JM, Korper W, Beertsen W. Cysteine proteinases and matrix metalloproteinases play distinct roles in the subosteoclastic resorption zone. J Bone Miner Res 1998; 13: 1420-1430.

19 Boissy P, Andersen TL, Abdallah BM, Kassem M, Plesner T, Delaissé JM. Resveratrol inhibits myeloma cell growth, prevents osteoclast formation, and promotes osteoblast differentiation. Cancer Res 2005; 65: 9943-9952.

20 Søe K, Delaissé JM. Glucocorticoids maintain human osteoclasts in the active mode of their resorption cycle. J Bone Miner Res 2010; 25: 2184-2192.

21 Gallagher JA. Human osteoblast culture. Methods Mol Med 2003; 80: 3-18.

22 Foged NT, Delaissé JM, Hou P et al. Quantification of the collagenolytic activity of isolated osteoclasts by enzyme-linked immunosorbent assay. J Bone Miner Res 1996; 11: 226-237.

23 Lund T, Søe K, Abildgaard N et al. First-line treatment with bortezomib rapidly stimulates both osteoblast activity and bone matrix deposition in patients with multiple myeloma, and stimulates osteoblast proliferation and differentiation in vitro. Eur J Haematol 2010; 85: 290-299.

24 Andreasen CM, Ding M, Overgaard S, Bollen P, Andersen TL. A reversal phase arrest uncoupling the bone formation and resorption contributes to the bone loss in glucocorticoid treated ovariectomised aged sheep. Bone 2015; 75: 32-39.

25 Gentzsch C, Delling G, Kaiser E. Microstructural classification of resorption lacunae and perforations in human proximal femora. Calcif Tissue Int 2003; 72: 698-709.

26 Mulari MT, Qu Q, Härkönen PL, Väänänen HK. Osteoblast-like cells complete osteoclastic bone resorption and form new mineralized bone matrix in vitro. Calcif Tissue Int 2004; 75: 253-261.

27 Jones SJ, Boyde A, Ali NN, Maconnachie E. A review of bone cell and substratum interactions - an illustration of the role of scanning electronmicroscopy. Scanning 1985; 7: 5-24.

28 Zhuo Y, Gauthier JY, Black WC, Percival MD, Duong LT. Inhibition of bone resorption by the cathepsin $\mathrm{K}$ inhibitor odanacatib is fully reversible. Bone 2014; 67: 269-280.

29 Drake FH, Dodds RA, James IE et al. Cathepsin K, but not cathepsins B, L, or S, is abundantly expressed in human osteoclasts. J Biol Chem 1996; 271: 12511-12516.

30 Tezuka K, Tezuka Y, Maejima A et al. Molecular cloning of a possible cysteine proteinase predominantly expressed in osteoclasts. J Biol Chem 1994; 269: 1106-1109.

31 Garnero P, Borel O, Byrjalsen I et al. The collagenolytic activity of cathepsin $\mathrm{K}$ is unique among mammalian proteinases. J Biol Chem 1998; 273: 32347-32352.

32 Delaisse JM, Boyde A, Maconnachie E et al. The effects of inhibitors of cysteine-proteinases and collagenase on the resorptive activity of isolated osteoclasts. Bone 1987; 8: 305-313.

33 Inui $\mathrm{T}$, Ishibashi $\mathrm{O}$, Inaoka $\mathrm{T}$ et al. Antisense oligodeoxynucleotide inhibits osteoclastic bone resorption. J Biol Chem 1997; 272: 8109-8112.

34 Parikka V, Lehenkari P, Sassi ML et al. Estrogen reduces the depth of resorption pits by disturbing the organic bone matrix degradation activity of mature osteoclasts. Endocrinology 2001; 142: 5371-5378. 
35 Susa M, Luong-Nguyen NH, Cappellen D, Zamurovic N, Gamse R. Human primary osteoclasts: in vitro generation and applications as pharmacological and clinical assay. J Transl Med 2004; $2: 6$.

36 Boyde A, Maconnachie E, Reid SA, Delling G, Mundy GR. Scanning electron microscopy in bone pathology: review of methods, potential and applications. Scan Electron Microsc 1986: 1537-1554.

37 Parfitt AM. Morphometry of bone resorption: introduction and overview. Bone 1993; 14: 435-441.

38 Jevon M, Sabokbar A, Fujikawa Y et al. Gender- and age-related differences in osteoclast formation from circulating precursors. J Endocrinol 2002; 172: 673-681.

39 Michelsen J, Wallaschofski H, Friedrich N et al. Reference intervals for serum concentrations of three bone turnover markers for men and women. Bone 2013; 57: 399-404.

40 Morgan JL, Heer M, Hargens AR et al. Sex-specific responses of bone metabolism and renal stone risk during bed rest. Physiol Rep 2014; 2(8). pii: e12119.

41 Minisola S, Dionisi S, Pacitti MT et al. Gender differences in serum markers of bone resorption in healthy subjects and patients with disorders affecting bone. Osteoporos Int 2002; 13: 171-175.

42 Doumouchtsis KK, Kostakis AI, Doumouchtsis SK et al. sRANKL/ osteoprotegerin complex and biochemical markers in a cohort of male and female hemodialysis patients. J Endocrinol Invest 2007; 30: 762-766.
43 Wang J, Stern PH. Sex-specific effects of estrogen and androgen on gene expression in human monocyte-derived osteoclasts. J Cell Biochem 2011; 112: 3714-3721.

44 Everts V, de Vries TJ, Helfrich MH. Osteoclast heterogeneity: lessons from osteopetrosis and inflammatory conditions. Biochim Biophys Acta 2009; 1792: 757-765.

45 Henriksen K, Bollerslev J, Everts V, Karsdal MA. Osteoclast activity and subtypes as a function of physiology and pathology--implications for future treatments of osteoporosis. Endocr Rev 2011; 32: 31-63.

46 Everts V, Korper W, Docherty AJ, Beertsen W. Matrix metalloproteinase inhibitors block osteoclastic resorption of calvarial bone but not the resorption of long bone. Ann N Y Acad Sci 1999; 878: 603-606.

47 Geblinger D, Geiger B, Addadi L. Surface-induced regulation of podosome organization and dynamics in cultured osteoclasts. Chembiochem 2009; 10: 158-165.

48 Duong LT. Therapeutic inhibition of cathepsin K-reducing bone resorption while maintaining bone formation. Bonekey Rep 2012; 1: 67.

(1) This work is licensed under a Creative Commons Attribution 4.0 Unported License. The images or other third party material in this article are included in the article's Creative Commons license, unless indicated otherwise in the credit line; if the material is not included under the Creative Commons license, users will need to obtain permission from the license holder to reproduce the material. To view a copy of this license, visit http:// creativecommons.org/licenses/by/4.0/

Supplemental Information for this article can be found on the Bone Research website (http://www.nature.com/boneres). 